\title{
Dieta de la población de guanacos (Lama guanicoe) reintroducida en el Parque Nacional Quebrada del Condorito, Argentina
}

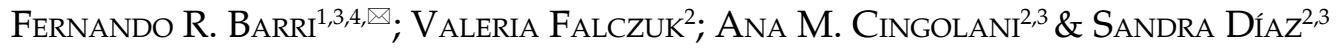 \\ 1. Centro de Ecología y Recursos Naturales Renovables, Universidad Nacional de Córdoba, Córdoba, Argentina. 2. Instituto \\ Multidisciplinario de Biología Vegetal (IMBIV-CONICET). 3. Departamento de Diversidad Biológica y Ecología, FCEFyN, \\ Universidad Nacional de Córdoba. 4. Instituto de Diversidad y Ecología Animal (IDEA-CONICET).
}

\begin{abstract}
Resumen. La reintroducción de especies silvestres es una herramienta recomendada cuando favorece la funcionalidad del ecosistema. Constituye un proceso a largo plazo durante el que es necesario evaluar los distintos aspectos ecológicos involucrados en la reintroducción. En el caso de los grandes herbívoros silvestres, un aspecto importante es conocer los hábitos de forrajeo. En el presente estudio se determinó la dieta a nivel de especie de la población de guanacos (Lama guanicoe) reintroducida en el Parque Nacional Quebrada del Condorito (Córdoba, Argentina). Para ello, entre febrero y agosto de 2009 se recolectaron muestras de heces frescas en del territorio ocupado por los grupos reproductivos dentro del Parque. La composición botánica de las muestras se determinó mediante análisis microhistológico. La dieta de los guanacos reintroducidos estuvo constituida principalmente por gramíneas y ciperáceas de bajo porte, características de los céspedes, y, a pesar de la alta diversidad de plantas vasculares presentes en el área de estudio, sólo cinco especies representaron entre 71\% (para los meses fríos y secos) y 93\% (para los meses cálidos y húmedos) de la dieta consumida durante el período de estudio: Sorghastrum pellitum, Chascolytrum subaristatum, Carex fuscula, Eleocharis pseudoalbibracteata y Lachemilla pinnata. Asimismo, las cuatro primeras especies fueron consumidas en una proporción significativamente mayor a la abundancia en el campo, lo que indicaría que los guanacos reintroducidos se comportaron como consumidores selectivos. Por otra parte, se detectó una variación estacional pequeña pero consistente en el consumo de las especies vegetales, incrementándose en los meses fríos y secos el consumo de las gramíneas de alto porte $D$. hieronymi y P. stuckertii, dominantes en los pajonales, y de especies leñosas. Es probable que esto se deba a la disminución de la productividad durante los meses fríos y secos en el área de estudio.
\end{abstract}

[Palabras clave: análisis microhistológico, herbivoría, Sierras Grandes del centro de Argentina]

\begin{abstract}
Diet of the reintroduced guanaco (Lama guanicoe) population into the Quebrada del Condorito National Park, Argentina: Wild species reintroduction is a highly recommended tool when favors ecosystem functionality. It constitutes a long-term process and requires the evaluation of the different ecological aspects involved in the reintroduction process. In the case of large wild herbivores one important aspect that should be known is foraging habits. In the present study, diet at the species level was determined for the guanaco (Lama guanicoe) population reintroduced in the Quebrada del Condorito National Park (Córdoba, Argentina). Fresh faeces samples were collected within the territory occupied by reproductive groups in the Park, between February and August 2009. Diet botanic composition was determined by microhistological analysis. The diet of reintroduced guanacos was composed mainly by short grasses and sedges, characteristic plants of grazing lawns. Despite the high plant diversity in the study area, only five species represented $71 \%$ (for the cold and dry months) to $93 \%$ (for the warm and wet months) of the diet consumed during the study period in the Park: Sorghastrum pellitum, Chascolytrum subaristatum, Carex fuscula, Eleocharis pseudoalbibracteata y Lachemilla pinnata. The former four species were consumed in a proportion significantly higher than their abundance in the field, which is indicating that the reintroduced guanacos are behaving as selective consumers. Additionally, a small but consistent seasonal difference was detected in plant species consumption, with higher consumption of the tussock grasses D. hieronymi and P. stuckertii, dominant in tussock grasslands, and of woody plants, during the dry and cold season. Probably this was caused by the productivity decrease during cold and dry season months in the study area.
\end{abstract}

[Keywords: herbivory, high mountains of central Argentina, microhistological analysis]

\section{INTRODUCCIÓN}

La reintroducción de especies silvestres es una herramienta muy utilizada en Biología de la Conservación, por lo que año a año se incrementa el número de proyectos de esta índole alrededor del mundo (Soorae 2011). Las reintroducciones son recomendadas cuando favorecen la funcionalidad del ecosistema y se realizan en un proceso a largo plazo

$\triangle$ fbarri@efn.uncor.edu
(Amstrong \& Seddon 2008). Sin embargo, reintroducir una especie es un proceso complejo que puede fracasar por falta del conocimiento necesario sobre la ecología de la especie y el sistema ecológico donde es introducida (Seddon et al. 2007). Por ejemplo, es importante establecer si en el sitio donde son liberados los individuos existen amenazas para su supervivencia (Bedin \& Ostrowski 1998), entender cómo los puede afectar la

Recibido: 19 de julio de 2013; Fin de arbitraje: 2 de noviembre; Última versión: 29 de enero de 2014; Aceptado: 27 de febrero. 
competencia interespecífica (Kaczensky et al. 2008) y conocer los requerimientos de hábitat y dieta de la población (Moser et al. 2006). Este tipo de información es necesario para diseñar las estrategias de manejo apropiadas que garanticen la supervivencia de los individuos y el éxito del proyecto de reintroducción (Soorae 2011). Por ello se destaca la importancia de evaluar científicamente los distintos aspectos ecológicos involucrados en el proceso de reintroducción (Amstrong \& Seddon 2008). En el caso de los herbívoros, la interacción con la vegetación es un proceso clave ya que las diferentes comunidades vegetales le permiten a los animales satisfacer sus requerimientos nutricionales y de hábitat (Godvik et al. 2009). Por lo tanto, dos aspectos relevantes que se deben estudiar son la selección de hábitat y la dieta de los individuos.

El cordón de las Sierras GrandesComechingones del centro de Argentina fue habitado por grandes herbívoros nativos durante el Holoceno, como el guanaco (Lama guanicoe), el venado de las pampas (Ozotoceros bezoarticus) y — probablemente- también la taruca (Hippocamelus antisensis), además de alguna de las dos especies de ñandú (Rhea sp.); todos se han extinguido localmente en el pasado reciente, entre los siglos XVIII y XX (Pastor \& Berberián 2007). Asimismo, hace alrededor de 400 años fue introducido el ganado doméstico, cuya acción sumada al uso del fuego como práctica de manejo de los pastizales ocasionó procesos erosivos, reducción de la cobertura vegetal y retracción de algunos bosques (Díaz et al. 1994; Renison et al. 2006; Cingolani et al. 2008; Cingolani et al. 2013).

En 1996 se creó el Parque Nacional Quebrada del Condorito, de 37340 ha. Desde ese momento, el ganado fue parcialmente excluido con el fin de evitar la degradación y favorecer la recuperación de la cobertura vegetal del área protegida. Esto aminoró o detuvo los procesos erosivos pero desencadenó una homogenización del paisaje dentro del Parque Nacional, en el que se observa un avance de los pajonales, dominados por Poa stuckertii (Hack.) Parodi, sobre los céspedes de alta diversidad (Cingolani et al. 2008; 2010). Por otro lado, en las áreas donde el ganado se mantuvo para evitar esta homogeneización los procesos erosivos no se detuvieron y la superficie de roca expuesta continúa en aumento (Cingolani et al. 2013). Por este motivo, la Administración de Parques Nacionales de Argentina tomó en 2007 la decisión de reintroducir al guanaco en el Parque, previo estudio de factibilidad (Tavarone et al. 2007), y siguiendo los protocolos internacionales para la reintroducción de especies de la vida silvestre (IUCN 1998).

Los grandes herbívoros están limitados fuertemente por alimento de alta calidad a lo largo de todo el año y, por lo tanto, seleccionan paisajes con abundancia de ítems forrajeros de mayor calidad nutricional (McNaughton 1986). En las Sierras Grandes de Córdoba, las plantas herbáceas bajas son los ítems que tienen mayores contenidos de nitrógeno, incluso en la estación seca, lo cual —en general- se asocia a una mejor calidad forrajera (Pucheta et al. 1998a; Vaieretti et al. 2013). Estas plantas incluyen gramíneas de bajo porte, ciperáceas, juncáceas y otras monocotiledóneas graminiformes y dicotiledóneas herbáceas. Muchas de estas especies son altamente consumidas por los herbívoros domésticos (Falczuk 2002) y a su vez son las principales componentes de los céspedes de pastoreo en el área de estudio (Cingolani et al. 2003; Vaieretti et al. 2010; 2013). Ello hace que los céspedes sean las comunidades más seleccionadas por los herbívoros domésticos y también por los guanacos (Flores et al. 2012; von Müller et al. 2012), a pesar de tener una productividad primaria neta aérea mucho menor en relación a los pastizales altos o "pajonales" (Pucheta et al. 1998b).

El guanaco es un herbívoro oportunista que, mediante adaptaciones anatómicas y fisiológicas, sobrevive en diferentes tipos de hábitat (González et al. 2006). Asimismo, en comparación con otros rumiantes, posee una buena capacidad para digerir plantas de calidad relativamente baja, lo que le permite alimentarse de una diversidad amplia de tipos de vegetación (Nugent et al. 2006). Por otra parte, el guanaco es un ramoneador de bajo impacto debido a la estructura digitígrada con almohadillas plantares de sus patas que evitan la compactación del suelo, al igual que la estructura de su boca que les permite seleccionar las partes de las plantas que consumen, sin arrancarlas de raíz; facilitando su rebrote (Erlich 1984). En términos de su conducta de forrajeo, el guanaco es considerado un herbívoro generalista de selectividad intermedia (Nugent et al. 2006). Si bien las variaciones observadas en los principales ítems alimentarios que consumen las poblaciones silvestres de guanaco dependen de las características del área y de las especies vegetales presentes en los diferentes 
sitios donde se estudió su dieta, en términos generales predominan las gramíneas (poáceas), seguidas en orden decreciente por otras especies similares a gramíneas (i.e., ciperáceas, juncáceas y otras monocotiledóneas), los arbustos y dicotiledóneas herbáceas y, en bajas proporciones, árboles, líquenes, epífitas y cactáceas (Bonino \& Pelliza Sbriller 1991; Candia \& Dalmaso 1995; Puig et al. 1996; Puig et al. 1997; Somlo et al. 1997; Cortés et al. 2006; Linares et al. 2010).

El proyecto de reintroducción del guanaco en las Sierras Grandes de Córdoba es el primer proyecto que intenta recuperar una especie silvestre extinta localmente dentro del Sistema Nacional de Áreas protegidas en Argentina. Por lo tanto, conocer cuáles son los ítems alimenticios consumidos por los guanacos en el Parque es relevante, tanto a los fines de evaluar si los individuos satisfacen sus requerimientos nutricionales como para sumar información que permita comprender el efecto que podría tener la herbivoría ejercida por la especie en el área protegida. En consecuencia, los objetivos de este trabajo fueron: 1) determinar y analizar la composición de la dieta de los guanacos reintroducidos en el Parque Nacional Quebrada del Condorito y su dinámica estacional, y 2) analizar la relación entre la variación estacional de la dieta y la disponibilidad en el campo de la vegetación consumida por los guanacos.

\section{MATERIALES y MÉTOdos}

\section{Área de estudio}

La porción superior de las Sierras Grandes de Córdoba, Argentina (1700 a 2900 m.s.n.m, 31³4' $\mathrm{S}, 64^{\circ} 50^{\prime} \mathrm{W}$ ) es una planicie con quebradas. Allí se ubica el Parque Nacional Quebrada del Condorito y la principal Reserva Hídrica de la región (Cingolani et al. 2004). La temperatura media del mes más cálido (enero) es $12.1^{\circ} \mathrm{C}$, la del mes más frío (julio) es $4.9^{\circ} \mathrm{C}$, y la media anual es $8.7^{\circ} \mathrm{C}$ (Colladon 2004). La precipitación media anual es $900 \mathrm{~mm}$ (Colladon 2010), concentrada entre octubre y abril (el promedio de este rango de meses es $828 \mathrm{~mm}$ ) (Figura 1). La productividad primaria neta aérea anual de los pastizales varía entre 404 y $830 \mathrm{~g} / \mathrm{m}^{2}$ según el tipo de comunidad. Debido al clima estacional, el crecimiento de la vegetación se concentra en los meses cálidos y húmedos, mientras que el resto del año la vegetación permanece inactiva (Pucheta et al. 1998b; Giorgis et al. 2010). Fitogeográficamente, el área pertenece al distrito chaqueño serrano (Cabrera 1976); no obstante, casi 50\% de su flora es de linaje andino y patagónico, y reúne además una diversidad rica en endemismos (Cabido et al. 1998). La vegetación se presenta como un mosaico heterogéneo, resultado de la interacción entre los factores topográficos y la historia de uso ganadero (Cingolani et al. 2008). Se pueden reconocer siete tipos principales de coberturas fisonómicas en el área (Cingolani et al. 2004; Flores et al. 2012): bosques y matorrales de Polylepis australis Bitter y Maytenus boaria Molina, pajonales gruesos de gramíneas de porte alto dominadas por $P$. struckertii, pajonales finos de gramíneas de porte alto dominados por Deyeuxia hieronymi (Hack.) Türpe y Festuca spp., céspedes secos dominados por especies herbáceas de bajo porte como Lachemilla pinnata (Ruiz \& Pav.) Rothm y Carex fuscula d'Urv. ssp. fuscula, céspedes húmedos con vegetación baja dominados por $L$. pinnata, C. fuscula y Eleocharis pseudoalbibracteata S. González \& Guagl, afloramientos rocosos naturales, y finalmente pavimentos y pedregales de roca desnuda por pérdida del suelo y cobertura vegetal.

El territorio utilizado por los guanacos reintroducidos en el Parque ocupa $49 \mathrm{~km}^{2}$ y se encuentra ubicado a 2100 metros de altura. Dentro de ese área, los pajonales gruesos ocupan la mayor parte de la superficie (24.9\%), continuando con los pavimentos y pedregales de erosión (19.5\%), los céspedes secos $(18.8 \%)$, los pajonales finos $(14.9 \%)$, los afloramientos rocosos $(11.2 \%)$, los bosques $(7 \%)$ y en último lugar los céspedes húmedos (3.9\%) (Flores et al. 2012).

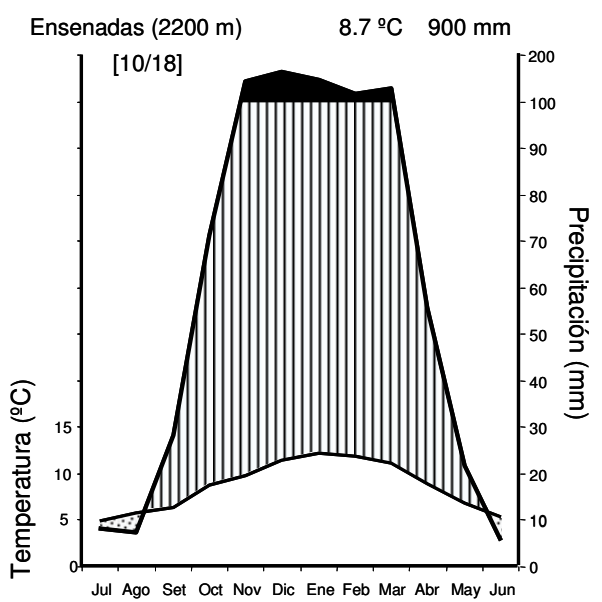

Figura 1. Climodiagrama de las Sierras Grandes de Córdoba, del Centro de Argentina, en las proximidades del área de estudio. El área punteada indica el período con déficit hídrico (curva de temperatura por encima de curva de precipitaciones), el área rayada el período con exceso hídrico (curva de precipitaciones por encima de curva de temperatura); el área pintada de negro indica el período con exceso hídrico en el que llueve más de 100 $\mathrm{mm} / \mathrm{mes}$.

Figure 1. Climate diagram of the high mountains of Córdoba, central Argentina, in the vicinity of the study area. The dotted area indicate the water deficit period (temperature curve above precipitation curve), the scratched area indicate the water excess period (precipitation curve above temperature curve); the black area indicate the water excess period with precipitation higher than $100 \mathrm{~mm} /$ month. 


\section{Muestreo y análisis de datos}

En el marco de un monitoreo continuo de la población de guanacos reintroducida (Barri et al. en prensa), entre febrero y agosto de 2009 se recorrió cada dos semanas el territorio utilizado por la población de guanacos. Las recorridas se realizaron tanto en camioneta como a caballo y a pie, y los grupos de guanacos fueron localizados mediante radiotelemetría. Al momento del estudio se encontraban establecidos en el Parque Nacional dos grupos reproductivos. En cada uno de los grupos, al menos un individuo contaba con radiocollar. Ambos grupos tenían áreas de acción definidas y diferenciadas entre sí (Flores et al. 2012). Un grupo estaba conformado por un macho, ocho hembras y dos juveniles, y el otro grupo por un macho y cuatro hembras. Durante las recorridas a campo se georeferenciaron los sitios de defecación grupal (bosteaderos) (Franklin 1982).

Durante febrero, marzo, abril, junio y agosto de 2009 (los tres primeros correspondientes al período cálido y húmedo y los dos últimos al período frío y seco del área de estudio) (Figura 1) se recolectaron 5 muestras mensuales de heces a partir de diferentes bosteaderos ( 25 muestras en total, en general de diferente bosteaderos, pero en algunos casos se repitieron entre fechas porque el total de bosteaderos en el área era menor a 25). Cada muestra estuvo constituida por el conjunto de tres colectas (submuestras) de heces frescas correspondientes a puntos cuadrantes opuestos entre sí de un mismo bosteadero, a los fines de minimizar la probabilidad de que las tres colectas correspondan a un mismo individuo. Las submuestras de heces fueron recogidas en bolsas de papel cartón y mantenidas en frío hasta su llegada al laboratorio. La composición botánica de las heces se determinó mediante un análisis microhistológico (Johnson et al. 1983) para el área de estudio. Las heces fueron secadas en estufa a $60{ }^{\circ} \mathrm{C} y$, con el objeto de disminuir los errores que pudieran resultar de la fragmentación diferencial, las submuestras fueron molidas en molinillo y tamizadas $(1 \mathrm{~mm})$ para producir fragmentos de tamaño relativamente uniforme. El material obtenido fue aclarado con hipoclorito de sodio al $50 \%$, y lavado con agua corriente sobre una malla fina para eliminar los fragmentos que no podían ser identificados por su tamaño reducido. Las submuestras, coloreadas con safranina al $1 \%$ en alcohol al $50 \%$, se montaron en gelatina glicerinada (preparados semipermanentes) y se observaron un total de 100 campos de microscopio por submuestra. Los fragmentos vegetales fueron identificados mediante la comparación con las descripciones de los caracteres epidérmicos de las especies documentadas por Falczuk et al. (1996). La identificación de los fragmentos vegetales se realizó hasta nivel de especie siempre que fue posible.

En primer término se calcularon las proporciones de cada una de las especies en las distintas muestras (se promedió - para cada caso- las proporciones de las tres submuestras por muestra). Luego se comparó el porcentaje de consumo entre fechas por medio de ANOVA de un factor, y en los casos en los que no se cumplieron los supuestos de normalidad y homocedasticidad, utilizando un análisis no paramétrico de Kruskall-Wallis $(N=5$ por fecha, Ntotal=25). Cuando se encontraron diferencias significativas en la proporción de especies consumidas entre fechas se hicieron comparaciones de a pares de Tukey o de MannWhitney según se cumplieran o no los supuestos, respectivamente.

Para analizar las tendencias generales en la variación dentro y entre fechas en la proporción de especies consumidas por los guanacos se realizó un análisis de componentes principales, utilizando las 25 muestras como casos y la proporción de todas las especies (o grupos de especies cuando no se pudo discriminar la especie) como variables. Luego se analizó si las cinco fechas diferían en su posición en los Ejes 1 y 2 del PCA por medio de ANOVA de un factor y comparaciones "a posteriori" de Tukey en el primer caso, y análisis de Kruskall-Wallis y comparaciones de Mann-Whitney en el segundo caso.

Por su parte, a partir de 47 censos de cobertura vegetal por especie realizados en la zona de estudio y en sus proximidades (Cabido 1985; Cingolani et al. 2003; 2010; Cingolani et al. datos no publicados) se calculó la cobertura relativa de todas las especies presentes en el territorio utilizado por los guanacos. Para ello, primero se calculó el promedio por especie para cada uno de los siete tipo de fisonomías, y luego se promedió entre fisonomías ponderando por la proporción del área que ocupa cada una (Flores et al. 2012). A partir de la cobertura, se calculó la proporción disponible por especie. Finalmente, se comparó la proporción de las especies en la dieta de los guanacos con la proporción disponible mediante el cálculo de los intervalos de confianza de los datos de dieta. Para hacer esta comparación, las muestras de las cinco fechas se agruparon "a posteriori", sobre la base de los resultados anteriores, en dos grandes grupos que representaban los dos períodos que diferían claramente entre sí (ver Resultados). En los casos en que los datos tenían muchos ceros y la distribución no se ajustaba a una distribución normal, los intervalos de confianza se calcularon utilizando 200 veces una selección de datos al azar (bootstrapping).

\section{Resultados}

Se registró un total de nueve especies vegetales en las muestras de heces de guanaco (Figura 2). Además, se detectó un grupo de poáceas no identificadas y un grupo de dicotiledóneas no identificadas, en ambos casos con baja proporción (Tabla 1). Las principales especies encontradas fueron las gramíneas de porte bajo Sorghastrum pellitum (Hack.) Parodi y Chascolytrum subaristatum (Lam.) Desv., las ciperáceas C. fuscula y E. 

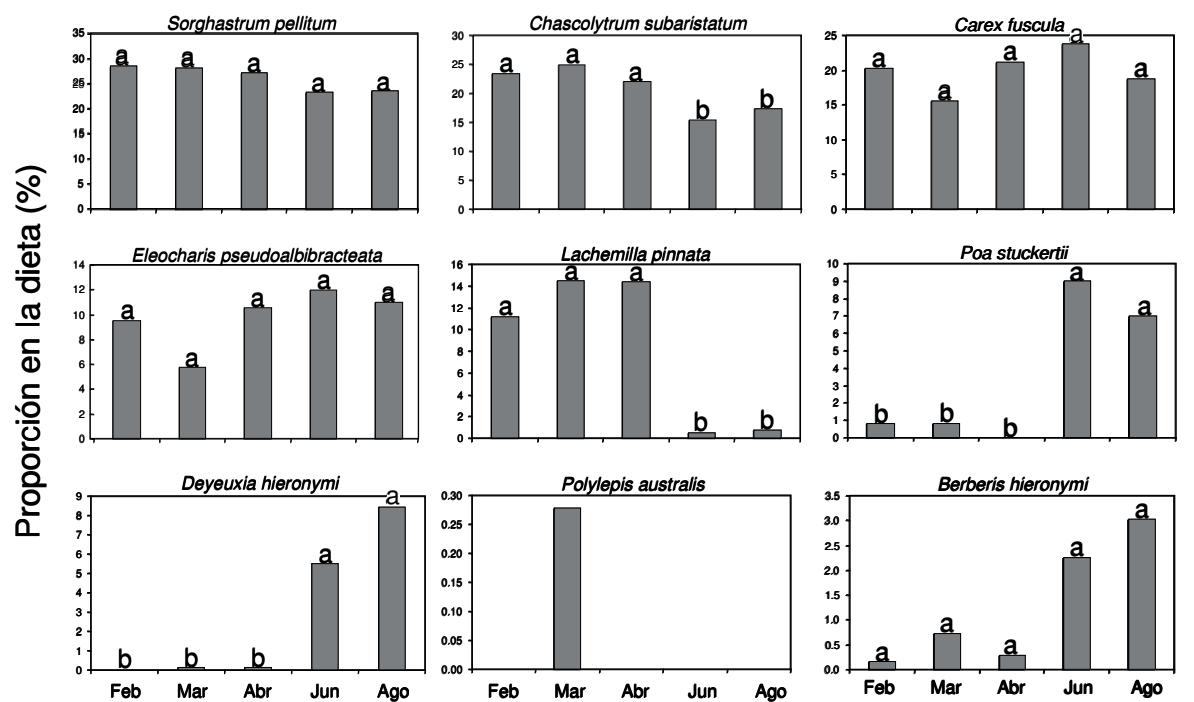

Figura 2. Proporción en la dieta, para las diferentes fechas de muestreo, de las nueve especies identificadas en las heces de los guanacos reintroducidos en el Parque Nacional Quebrada del Condorito (notar que la escala es diferente en los distintos gráficos para resaltar las variaciones entre meses para las distintas especies). Letras distintas indican diferencias significativas $(P \leq 0.05)$, para el caso de Polylepis australis no se hizo estadística porque estuvo presente en una sola muestra.

Figure 2. Proportion in the diet for different sampling dates, of the nine species identified in the feces of guanacos reintroduced into the Quebrada Condorito National Park (note that the scale is different in the various charts to highlight variations between months for different species). Different letters indicate significant differences $(\mathrm{P} \leq 0.05)$, for the case of Polylepis australis was not done because statistics was present in a single sample.

pseudoalbibracteata, y la dicotiledónea herbácea L. pinnata. En conjunto, estas cinco especies representaron entre 71 y $93 \%$ de la dieta consumida durante el período de estudio en el Parque. Gramíneas de porte alto como
P. stuckertii y D. hieronymi se encontraron en una proporción no superior al 16\% (Figura 2). También se encontraron, aunque en muy baja proporción, el arbusto Berberis hieronymi C.K. Schneid y el árbol $P$. australis.

Tabla 1. Disponibilidad promedio e intervalos de confianza de las especies vegetales consumidas por los guanacos reintroducidos en el Parque Nacional Condorito, durante el período cálido y húmedo (febrero, marzo y abril) y el período frío y seco (junio y agosto). En la última columna se indica la disponibilidad en el territorio (Flores et al. 2012).

Table 1. Average percentage and confidence intervals of the plant species consumed by reintroduced guanacos in Quebrada del Condorito the National Park, during the warm and wet period (February, March and April) and cold and dry period (June to August). In the last column the territory availability is indicated (Flores et al. 2012).

\begin{tabular}{|c|c|c|c|c|}
\hline \multirow[t]{2}{*}{ Forma de vida } & \multirow[t]{2}{*}{ Especie } & \multicolumn{2}{|c|}{ Consumo (\%) e intervalo de confianza } & \multirow{2}{*}{$\begin{array}{c}\text { Disponibilidad } \\
(\%)\end{array}$} \\
\hline & & Meses cálidos y húmedos & Meses fríos y secos & \\
\hline Gramíneas de porte bajo & Sorghastrum pellitum & $\begin{array}{c}27.8 \\
(25.1-30.6)\end{array}$ & $\begin{array}{c}23.3 \\
(17.6-28.9)\end{array}$ & 1.2 \\
\hline & $\begin{array}{l}\text { Chascolytrum } \\
\text { subaristatum }\end{array}$ & $\begin{array}{c}23.4 \\
(20.8-26.0)\end{array}$ & $\begin{array}{c}16.3 \\
(13.2-19.35)\end{array}$ & 0.1 \\
\hline Ciperáceas de porte bajo & Carex fuscula & $\begin{array}{c}19.0 \\
(15.8-22.1)\end{array}$ & $\begin{array}{c}21.3 \\
(16.6-26.0)\end{array}$ & 3.9 \\
\hline & $\begin{array}{l}\text { Eleocharis } \\
\text { pseudoalbibracteata }\end{array}$ & $\begin{array}{c}8.6 \\
(6.0-11.1)\end{array}$ & $\begin{array}{c}11.5 \\
(9.5-13.4)\end{array}$ & 0.7 \\
\hline $\begin{array}{l}\text { Dicotiledónea herbácea } \\
\text { rastrera }\end{array}$ & Lachemilla pinnata & $\begin{array}{c}13.3 \\
(10.4-16.3)\end{array}$ & $\begin{array}{c}0.6 \\
(0.1-1.1)\end{array}$ & 13.9 \\
\hline Gramíneas de porte alto & Poa stuckertii & $\begin{array}{c}0.5 \\
(0.1-1.0)\end{array}$ & $\begin{array}{c}8.0 \\
(5.3-10.6)\end{array}$ & 19.4 \\
\hline & Deyeuxia hieronymi & $\begin{array}{c}0.1 \\
(0.0-0.3)\end{array}$ & $\begin{array}{c}7.0 \\
(4.9-9.1)\end{array}$ & 22.1 \\
\hline Árbol & Polylepis australis & $\begin{array}{c}0.1 \\
(0.0-0.3)\end{array}$ & 0 & 4.9 \\
\hline Arbusto & Berberis hieronymi & $\begin{array}{c}0.4 \\
(0.0-0.7)\end{array}$ & $\begin{array}{c}2.6 \\
(1.5-3.9)\end{array}$ & 0.4 \\
\hline Gramínea de porte bajo & Poácea N/I & 4.5 & 9.2 & - \\
\hline $\begin{array}{l}\text { Dicotiledónea herbácea } \\
\text { rastrera }\end{array}$ & Dicotiledónea N/I & 2.3 & 0.3 & - \\
\hline
\end{tabular}


Por otra parte, los guanacos reintroducidos variaron la proporción en que consumen las diferentes especies a lo largo del año (Figura 2). C. subaristatum y L. pinnata fueron consumidas en una proporción significativamente mayor en los tres meses más cálidos y húmedos (febrero, marzo y abril) en relación a los dos meses más fríos y secos (junio y agosto). Asimismo, las especies de porte alto $D$. hieronymi y $P$. stuckertii siguieron el patrón opuesto, con un consumo casi nulo en los meses cálidos y húmedos, y un consumo significativamente mayor en los meses fríos y secos. El resto de las especies no difirieron significativamente entre estaciones, pero se observó una tendencia del arbusto $B$. hieronymi a ser más consumido en los meses fríos y secos $(P=0.08)$. Dado que los resultados mostraron que los patrones de la dieta forman dos grupos homogéneos [la dieta de los meses más cálidos y húmedos (febrero, marzo, abril) y - por otro lado- la dieta de los meses más fríos y secos (junio y agosto)], los promedios y los intervalos de confianza de la proporción del consumo de cada ítem forrajero se calcularon para cada uno de estos dos grupos (Tabla 1).

Estas tendencias estacionales se reflejaron en el análisis de componentes principales (Figura 3), donde el Eje 1 separó con claridad en el extremo negativo las muestras con mayor proporción de gramíneas y dicotiledóneas de porte bajo, colectadas en los meses de febrero, marzo y abril, de las muestras con una mayor proporción de gramíneas de porte alto. Estas últimas muestras, correspondientes a junio y agosto, se ubicaron en el extremo positivo del Eje 1. El análisis de varianza y las comparaciones "a posteriori" indicaron que los tres primeros meses (febrero, marzo, abril), más húmedos y cálidos, forman un conjunto estadísticamente homogéneo y significativamente diferente de los dos últimos meses (junio y agosto), más secos y fríos (Figura 1). Junio y agosto formaron un conjunto homogéneo entre sí $(F=17.58, P \leq$ 0.05). Por otro lado, el Eje 2 separó las muestras con mayor proporción de leñosas en el extremo positivo respecto de las muestras con menor proporción de leñosas y mayor proporción de ciperáceas, en el extremo negativo. La prueba de Kruskall-Wallis mostró leves diferencias entre fechas $\left(\mathrm{Chi}^{2}=9.77, P=0.044\right)$; el mes de agosto, con posiciones más altas en el Eje 2 , se diferenció de los meses de febrero y abril, con posiciones más bajas en el Eje 2 (Pruebas de Mann-Whitney, $P \leq 0.05)$. Los meses de marzo y junio no se diferenciaron significativamente de ninguna de las demás fechas (Pruebas de Mann-Whitney, $P \geq 0.05$ ) (Figura 3).

De los censos utilizados para evaluar la cobertura de especies en el territorio utilizado por los guanacos se observó que existe un total de 183 especies en el área de estudio. Los resultados del análisis comparativo indican que S. pellitum, C. subaristatum, C. fuscula y E. pseudoalbibracteata, todas gramíneas y
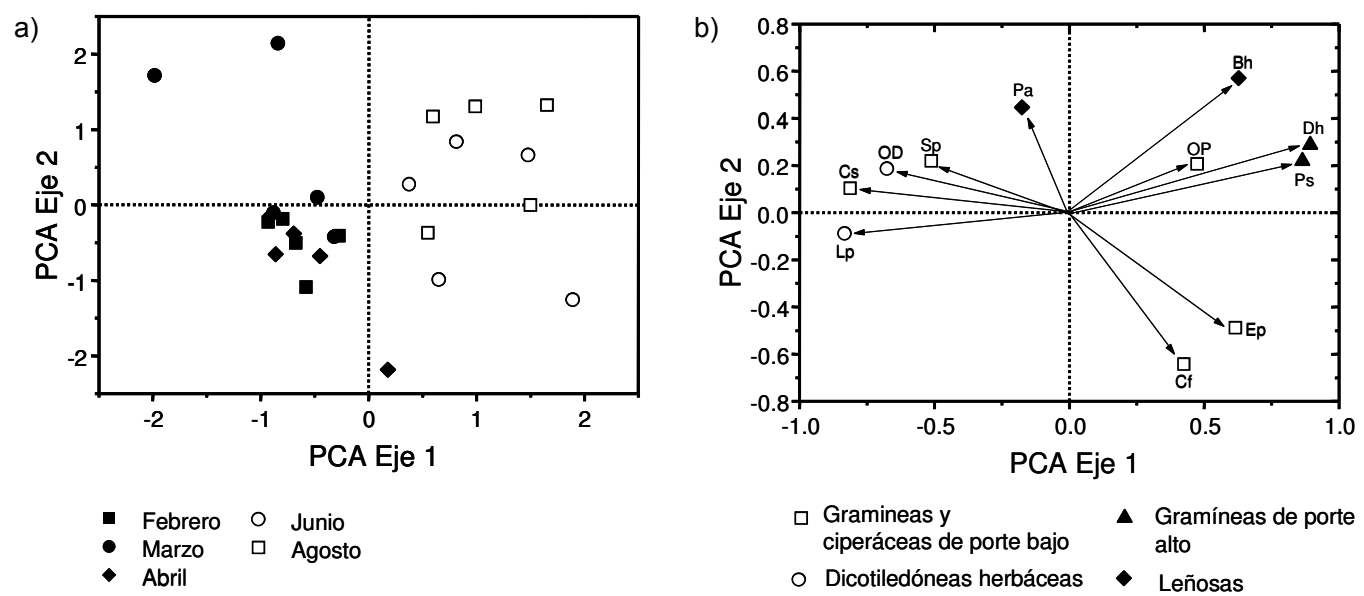

Figura 3. Ubicación de las muestras (a) y contribución de las especies (b) en los dos primeros Ejes del Análisis de Componentes Principales. En (a) los diferentes símbolos indican las distintas fechas de muestreo, y en (b) indican diferentes formas de vida. Sp: Sorghastrum pellitum, Cs: Chascolytrum subaristatum, Cf: Carex fuscula, Ep: Eleocharis pseudoalbibracteata, Lp: Lachemilla pinnata, Op: Poaceas N/I, Ps: Poa stuckertii, Dh: Deyeuxia hieronymi, Od: Dicotiledóneas N/I, Bh: Berberis hieronymi, Pa: Polylepis australis.

Figure 3. Location of the samples (a) and contribution of species (b) in the first two axes of the Principal Component Analysis. In (a) the different symbols indicate the different sampling dates, and (b) show different ways of life. Sp: Sorghastrum pellitum, Cs: Chascolytrum subaristatum, Cf: Carex fuscula, Ep: Eleocharis pseudoalbibracteata, Lp: Lachemilla pinnata, Op: Poaceas N/I, Ps: Poa stuckertii, Dh: Deyeuxia hieronymi, Od: Dicotiledóneas N/I, Bh: Berberis hieronymi, Pa: Polylepis australis. 
ciperáceas de porte bajo, fueron consumidas en una proporción significativamente mayor a su disponibilidad en el campo, tanto en el período cálido y húmedo como en el frío y seco. Por otro lado, las gramíneas de porte alto $D$. hieronymi y $P$. stuckertii fueron consumidas en menor proporción a lo disponible para ambos períodos. Por su parte, L. pinnata y B. hieronymi fueron consumidas en una proporción similar a lo disponible durante el período cálido y húmedo, en tanto que en el período frío y seco la primera fue menos consumida y la segunda más consumida en relación a lo disponible en el campo (Tabla 1).

\section{DisCUSIÓN}

La dieta de los guanacos reintroducidos en el Parque Nacional Quebrada del Condorito estuvo constituida principalmente por gramíneas y herbáceas de bajo porte, en tanto las gramíneas de porte alto quedaron relegadas a porcentajes bajos y casi exclusivamente en el período invernal. Del total de 183 especies de plantas vasculares presentes en el área de estudio, sólo cuatro (L. pinnata, C. subaristatum, C. fuscula y E. pseudoalbibracteata) representaron cerca de $80 \%$ de la dieta en los meses cálidos y húmedos, y algo más de $70 \%$ en los meses fríos y secos, además de haber sido consumidas en una proporción muy superior a su disponibilidad en el campo, para ambos períodos. A su vez, estas especies vegetales son las más abundantes en los céspedes de la región, lo que coincide con los resultados observados por Flores et al. (2012), quienes determinaron que los céspedes, tanto húmedos como secos, fueron las unidades de hábitat seleccionadas por sobre su disponibilidad en el territorio utilizado por los grupos de guanacos establecidos en el Parque. Por lo tanto, se puede suponer que los guanacos reintroducidos se comportaron como consumidores selectivos (Linares et al. 2010).

El patrón observado de consumo de gramíneas y ciperáceas de porte bajo por sobre las gramíneas de porte alto también ha sido observado en vacas y caballos para las Sierras Grandes deCórdoba(Falczuk 2002). Ello puede deberse a que en este ecosistema de montaña existe una limitación fuerte de alimento de alta calidad nutritiva, y las gramíneas y ciperáceas bajas son las que presentan los mayores contenidos de nitrógeno (Pucheta et al. 1998a). Estas formas de vida, aunque no son muy abundantes en las comunidades dominantes del área de estudio (cubierta en su mayor parte por pajonales gruesos y finos), son las dominantes en los céspedes (Cingolani et al. 2003). Ello explicaría, por ejemplo, que $P$. stuckertii —especie endémica que cubre la mayor parte del territorio ocupado por los guanacos reintroducidos formando pajonales altos y densos (Flores et al. 2012) — no fuera una especie consumida, en tanto que especies de gramíneas bajas mucho menos abundantes, como C. subaristatum y $S$. pellitum, resultaron muy consumidas.

Por su parte, se destaca la variación estacional en el consumo de las especies vegetales encontradas en las muestras de heces recogidas en el período de estudio. Es evidente que durante el período cálido y húmedo —el más productivo en este ecosistema (Pucheta et al. 1998b) - los guanacos se alimentan sobre todo de las especies de pastos bajos, mientras que en el invierno se incrementa el consumo de gramíneas presentes en los pajonales, afloramientos rocosos o bosques (e.g., D. hieronymi y otros ítems alimenticios como arbustos y árboles). Ello indicaría que la disminución invernal de la productividad en el área de estudio obliga a los guanacos a consumir una mayor proporción de recursos alimenticios menos palatables o nutritivos, pero más abundantes, con el fin de asegurarse sus requerimientos energéticos. El guanaco es una especie capaz de modificar su dieta estacionalmente de acuerdo a la disponibilidad en el ambiente, lo cual le otorga una flexibilidad dietaria elevada (Puig et al. 2001). En el caso de las ciperáceas (e.g., E. pseudoalbibracteata, especie muy palatable), el consumo es levemente superior en invierno, lo que tal vez se deba a que en el verano esta especie se encuentra menos disponible debido al anegamiento de los céspedes húmedos, donde es común encontrarla. Por su parte, aunque fue registrado en una proporción muy baja $(0.05 \%)$, es importante destacar la presencia de $P$. australis en las muestras de heces del invierno dado que el ganado doméstico consume entre 65\% (a cargas moderadas) y $85 \%$ (a cargas altas) de la productividad anual de los individuos de esta especie menores a $2 \mathrm{~m}$ de altura (Giorgis et al. 2010).

Si bien en el área de estudio los guanacos presentan patrones similares al ganado doméstico en lo que respecta a la selección de hábitat (Flores et al. 2012; von Müller et al. 2012) y estacionalidad de la dieta (Falczuk 2002), podrían presentar algunas diferencias en los patrones de forrajeo. Un 
ejemplo de ello es el consumo importante de Lachemilla pinnata por parte de los guanacos reintroducidos; aunque es una planta común y de alto valor forrajero, por su hábito de crecimiento rastrero es poco consumida por vacas y caballos (Falczuk 2002). Por su parte, a pesar de que las especies más consumidas por los guanacos coinciden con algunas de las más seleccionadas por el ganado doméstico criado en las Sierras Grandes de Córdoba, el número de especies vegetales encontradas en las heces de los guanacos reintroducidos es menor al registrado en heces de vacas y caballos, donde aparecen otras especies del área de estudio (e.g., Agrostis spp., Sporobolus indicus (L.) R. Br. var. indicus, Juncus pallescens Lam. var. achalensis (Barros) Novara y Mulhenbergia peruviana (P. Beauv.) Steud (Falczuk 2002)). Sin embargo, al haberse realizado este estudio un año después de ser liberados los individuos, el comportamiento de forrajeo de los guanacos en el área deberá corroborarse con un estudio comparativo respectodelganadodoméstico;en el cúal se deberán establecer clausuras en áreas homogéneas para los diferentes herbívoros, que - en cada caso - permitan evaluar a largo plazo la dinámica de la vegetación y de las interacciones planta-animal.

Tanto el presente estudio como el realizado por Flores et al. (2012) permiten establecer que la disponibilidad de forraje de alto valor nutritivo (que en las Sierras Grandes de Córdoba se encuentra representado por las gramíneas y ciperáceas bajas) explica en gran medida la selección de hábitat y también de la dieta de los guanacos reintroducidos en el Parque Nacional Quebrada del Condorito. Esta información tiene relevancia para este importante proyecto de conservación y restauración ecológica encarado por la Administración de Parques Nacionales, dado que - junto con los restantes estudios desarrollados hasta el momento (Barri \& Fernández 2011; Flores et al. 2012; Barri \& Cufré, en prensa) - permitirá tomar las medidas de manejo más apropiadas para garantizar su éxito. Asimismo, será de suma utilidad estudiar la hipótesis referida a si la selección de hábitat y dieta que realizan los guanacos en el Parque podrán contribuir a mantener los céspedes de alta diversidad florística en el área protegida, evitando así el avance de los pajonales sobre los mismos.

Agradecimientos: a las autoridades y personal del Parque Nacional Quebrada del Condorito por permitirnos realizar el trabajo de campo y la recolección de las muestras, en particular a G. Jaacks por impulsar el Proyecto de reintroducción del guanaco. Este estudio fue financiado por la Secretaría de Ciencia y Técnica (SECyT) de la Universidad Nacional de Córdoba, por el Consejo Nacional de Investigaciones Científicas y Técnicas (CONICET) de Argentina, y por el Instituto Interamericano de Investigaciones sobre el Cambio Global (IAI) (subsidios CRN 2015 y SGP-CRA2015, correspondientes a los subsidios GEO-0452325 and GEO1138881 de la US National Science Foundation).

\section{BIBLIOGRAFÍA}

AMStrong, P \& P SEDDON. 2008. Directions in reintroduction biology. Trends Ecol. E Evol., 23:20-25.

BARRI, F \& M CUFRÉ. En prensa. Supervivencia de Guanacos (Lama guanicoe) reintroducidos con y sin período de pre-adaptación en el Parque Nacional Quebrada del Condorito, Argentina. Mastozoología Neotropical.

Bedin, E \& S OstrowsKi. 1998. Arabian oryx (Oryx leucoryx) reintroductions in Saudi Arabia: update. Newsletter of the IUCN Re-introduction Specialist Group, 16:13-14.

Bonino, N \& A Pelliza Sbriller. 1991. Composición Botánica de la dieta del guanaco en dos ambientes contrastantes de Tierra del Fuego, Argentina. Ecol. Aust., 1:97-102.

CABIDO, M. 1985. Las comunidades vegetales de la Pampa de Achala, Sierras de Córdoba, Argentina. Documents Phytosociologiques, 9:431-443.

CABido, M; G Funes; E PuCheta; F VendRAMINI \& S DíAz.1998. A chorological analysis of the mountains from central Argentina: is all what we call Sierra Chaco really Chaco? Contribution to the study of the flora and vegetation of the Chaco XII. Candollea, 53:321-331.

Cabrera, AL. 1976. Regiones Fitogeográficas argentinas. 2 ed. EnCIClop. Arg. Agric. Y Jardinería. ACME (ed.), Buenos Aires.

CAndia, R \& AD Dalmasso. 1995. Dieta del guanaco (Lama guanicoe) y productividad del pastizal en la reserva $\mathrm{La}$ Payunia, Mendoza (Argentina). Multequina, 4:5-15.

Cingolani, AM; M Cabido; D Renison \& V Solís NefFa. 2003. Combined effects of environment and grazing on vegetation structure in Argentine granite grasslands. J. Veg. Sci., 14:223-232.

Cingolani, AM; D Renison; M ZaK \& M Cabido. 2004. Mapping vegetation in a heterogeneous mountain rangeland using landsat data: an alternative method to define and classify land-cover units. Remote Sens., 94:84-97.

Cingolani, AM; D Renison; P Tecco; DE Gurvich \& M CABIDO. 2008. Predicting cover types in a mountain range with long evolutionary grazing history: a GIS approach. J. Biogeog., 35:538-551.

Cingolani, AM; MV VaieretTi; DE Gurvich; M Giorgis \& M CABIDO. 2010. Predicting alpha, beta and gamma plant diversity from physiognomic and physical indicators as a tool for ecosystem monitoring. Biol. Conserv., 143: 2570-2577.

Cingolani, AM; MV Vaieretti; M Giorgis; N La Torre; J Whitworth-Hulse; ET AL. 2013. Can livestock and fires convert the sub-tropical mountain rangelands of central Argentina into a rocky desert? The Range. J., 35:285-297

Colladon, L. 2004. Anuario de temperaturas medias 19942003. Cuenca del Río San Antonio. Sistema del Río Suquía - Provincia de Provincia de Córdoba. ('Annual ploviometry 1992-2010 - San Antonio river. Suquía river system Córdoba Province.'). Instituto Nacional del Agua y del Ambiente (INAA) y Centro de Investigaciones de la Región Semiárida (CIRSA): Córdoba, Argentina. 
Colladon, L. 2010. Anuario pluviométrico 1992-2010. Cuenca del Río San Antonio. Sistema del Río Suquía - Provincia de Provincia de Córdoba. ('Annual ploviometry 1992-2010 - San Antonio river. Suquía river system - Córdoba Province.'). Instituto Nacional del Agua y del Ambiente (INAA) y Centro de Investigaciones de la Región Semiárida (CIRSA): Córdoba, Argentina.

Cortés, A; E Miranda \& F López-Cortés. 2006. Abundancia y dieta del camélido Lama guanicoe en un ambiente altoandino del norte-centro de Chile. Pp. 383-411 en: Cépeda, PJ (ed.). Geoecología de los andes desérticos: la Alta Montaña del Valle del Elqui. La Serena, Chile.

Díaz, S; A ACOSTA \& M CABIDO. 1994. Community structure in montane grasslands of central Argentina in relation to land use. J. Veg. Sci., 5:483-488.

ERlich, A. 1984. Guanaco. Pp. 274-283 en: Centro Editor de América Latina (eds.). Mamíferos. Buenos Aires, Argentina.

Falczuk, V; VR Rosati \& M Cabido. 1996. Caracteres epidérmicos foliares de valor diagnóstico para identificar especies vegetales de Pampa de Achala (Córdoba, Argentina) Agriscientia, 13:41-58. Disponible en: http://www.efn.unc.edu.ar/otros/bibliocentro/ TesBio.htm

FALCZuK, V. 2002. Relaciones entre selección de dieta de grandes herbívoros, disponibilidad y características físico-químicas de las plantas en un pastizal de altura de las Sierras de Córdoba. Tesis Doctoral. Universidad Nacional de Córdoba. Argentina.

Flores, C; AM Cingolani; A Von Müller \& FR Barri. 2012. Habitat selection by reintroduced guanacos (Lama guanicoe) in a heterogeneous mountain rangeland of central Argentina. The Range. J., 34:439-445.

FRANKLIN, WL. 1982. Biology, ecology, and relationship to man of the South American camelids Special Publication Series Vol. 6. Pp 457-489 en: Mares, ML \& H Genoway (eds.). Mammalian biology in South America. University of Pittsburgh, USA.

Giorgis, MA; AM Cingolani; I TeICH; D Renison \& I HenSEN. 2010. Do Polylepis australis trees tolerate herbivory? Seasonal patterns of shoot growth and its consumption by livestock. Plant Ecol., 207:307-319.

González, BA; RE Palmas; B Zapata \& JC Marín. 2006. Taxonomic and biogeographical status of guanaco Lama guanicoe (Artiodactyla, Camelidae). Mammal Rev., 36:157-178.

IUCN. 1998. The IUCN Guidelines for Re-introductions. IUCN/SSC Reintroductions Specialist Group. Cambridge, UK. Pp. 18.

Johnson, MK; H Wofford \& HA Pearson. 1983. Digestion and fragmentation. Influence on herbivore diet analysis. J. Wildl. Manage., 47:877- 879.

KaCZensKy, P; O GanbaAtar; H Von Wehrden \& C Walzer. 2008. Resource selection by sympatric wild equids in the Mongolian Gobi. J. Appl. Ecol., 45:1762-1769.

Linares, L; V Linares; G Mendoza; F Peláez; E Rodríguez; ET AL. 2010. Preferencias alimenticias del guanaco (Lama guanicoe cacsilensis) y su competencia con el ganado doméstico en la Reserva Nacional de Calipuy, Perú. Scientia Agropecuaria, 1:225-234

McNaughton, SJ. 1986. Grazing Lawns: On Domesticated and Wild Grazers. Amer. Nat., 128:937-939.

Moser, B; M SchÜtz \& KE Hinderlang. 2006. Importance of alternative food resource for browsing by roe deer on deciduous trees: the role the food availability and species quality. Forest Ecol. Manag., 226:248-255.

Nugent, P; R Baldi; P Carmanchahi; D De Lamo; M Failla; ET AL. 2006. Conservación del guanaco en Argentina. Pp: 137-149 en: Bolkovic, ML y D Ramadori (eds.). Manejo de Fauna Silvestre en la Argentina. Programas de uso sustentable. Dirección de Fauna Silvestre, Secretaría de Ambiente y Desarrollo Sustentable. Buenos Aires, Argentina.

Pastor, S \& E Berberián. 2007. Arqueología del sector central de las Sierras de Córdoba (Argentina). Hacia una definición de los procesos sociales del período prehispánico tardío (900-1573 d.C.). Intersecciones en Antropología, 8:31-49.

Pucheta, E; F Vendramini; M Cabido \& S. Díaz. 1998a. Estructura y funcionamiento de un pastizal de montaña bajo pastoreo y su respuesta luego de su exclusión. Revista de la Facultad de Agronomía de La Plata, 103: 77-92.

Pucheta, E; M Cabido; S Díaz \& G. Funes. 1998b. Floristic composition, biomass, and above ground net plant production in grazed and protected sites in a mountain grassland of central Argentina. Acta Oecol., 19:97-105.

Puig, S; F Videla; SA Monge \& V Roig. 1996. Seasonal variations in guanaco diet (Lama guanicoe Müller 1776) and food availability in Northern Patagonia, Argentina. J. Arid Envir., 34:215-224.

PUIG, S; F VIDELA \& MI Cona. 1997. Diet and abundance of the guanaco (Lama guanicoe Müller 1776) in four habitats of northern Patagonia, Argentina. J. Arid Envir., 36:343-357.

Puig, S.; F Videla; M Cona; S Monge. 2001. Use of food availability by guanacos (Lama guanicoe) and livestock in Northern Patagonia (Mendoza, Argentina). J. Arid Envir., 47:291-308.

Seddon, P; P Armstrong \& RF Maloney. 2007. Developing the Science of Reintroduction Biology. Conserv. Biol., 21: 303-312.

Somlo, R; G Bonvissuto; M Mancora; A Pelliza Sbriller; P WILleMs; ET AL. 1997. Atlas dietario de los herbívoros patagónicos. EEA INTA Bariloche, Argentina. Pp. 64.

SOORAE, PS. 2011. Global Re-introduction Perspectives: 2011. More case studies from around the globe. IUCN/SSC Reintroduction Specialist Gland, Switzerland. Pp. 250.

Tavarone, EG; L Ruíz; J Monguillot \& D Ramírez. 2007. Informe de Avance. Proyecto piloto de reintroducción del guanaco (Lama guanicoe) en el Parque Nacional Quebrada del Condorito (PNQC). Delegación Regional Centro de la Administración de Parques Naciones, Argentina.

Vaieretti, MV; AM Cingolani; N Pérez Harguindeguy; DE GuRvich \& M CABIDO. 2010. Does decomposition of standard materials differ among grassland patches maintained by livestock?. Austral Ecol., 35:935-943.

Vaieretti, MV; AM Cingolani; N Pérez Harguindeguy \& M CABIDO. 2013. Effects of differential grazing on decomposition rate and nitrogen availability in a productive mountain grassland. Plant and Soil, 371 : 675-691.

Von Müller, A; Cingolani, AM; MV Vaieretti; M \& D RENISON. 2012. Estimación de carga bovina localizada a partir de frecuencia de deposiciones en un pastizal de montaña. Ecología Austral, 22:178-187. 